\title{
Oculocutaneous albinism type 6
}

INSERM

\section{Source}

INSERM. (1999). Orphanet: an online rare disease and orphan drug data base.

Oculocutaneous albinism type 6. ORPHA:370097

Oculocutaneous albinism type 6 (OCA6) is a type of oculocutaneous albinism, recently discovered in one Chinese family, characterized by light hair at birth that darkens with age, white skin, transparent irides, photophobia, nystagmus, foveal hypoplasia and reduced visual acuity and that is due to mutations in the SLC24A5 gene (15q21.1). 